\title{
Erratum to: Effect of burn-up on the radioactivation behavior of cladding hull materials studied using the ORIGEN-S code
}

\author{
Min Ku Jeon - Chang Hwa Lee $\cdot$ Chang Je Park • \\ Jung Hoon Choi · In Hak Cho · Kweon Ho Kang • \\ Hwan-Seo Park · Geun II Park
}

Published online: 20 December 2013

(C) Akadémiai Kiadó, Budapest, Hungary 2013

\section{Erratum to: J Radioanal Nucl Chem (2013) 298:1629-1633 \\ DOI 10.1007/s10967-013-2543-6}

Unfortunately in the original publication some errors were found in the input data of simulation program. Consequently, the values of Tables 2, 3, 4, and 5 and Fig. 1 have been changed and the revised version of tables and figures are given in the following pages. In the original work, the authors have mentioned an increase of total radioactivity which was about 16.7-24\% when fuel burn-up increased from 30 to 75 GWD/MTU. But it should be noted that in the new calculation results, a significant increase in the radioactivity was observed between 30 and 45 GWD/MTU, and the total radioactivity values increased by 167,262 , 280, and $318 \%$ for the Zry-4, Zirlo, HANA-4, and HANA6 cases, respectively, when the fuel burn-up increased from 30 to $75 \mathrm{GWD} / \mathrm{MTU}$. Although the values were changed, the conclusion for the view point of ${ }^{94} \mathrm{Nb}$ and ${ }^{60} \mathrm{Co}$ still remains same as shown in the new Fig. 1.

The online version of the original article can be found under doi:10.1007/s10967-013-2543-6.

M. K. Jeon $(\bowtie) \cdot$ C. H. Lee $\cdot$ J. H. Choi ·

I. H. Cho - K. H. Kang - H.-S. Park - G. I. Park

Nuclear Fuel Cycle Waste Treatment Research Division, Korea

Atomic Energy Research Institute, 989-111 Daedeok-daero,

Yuseong-gu, Daejeon 305-353, Republic of Korea

e-mail: minku@kaeri.re.kr

C. J. Park

Reactor Core Design Division, Korea Atomic Energy Research

Institute, 989-111 Daedeok-daero, Yuseong-gu,

Daejeon 305-353, Republic of Korea 
Table 2 The radioactivation calculation results of Zircaloy-4 for various burn-ups of 30, 45, 60, and 75 GWD/MTU (only major nuclides are shown)

\begin{tabular}{lllll}
\hline & 30 GWD/ & $45 \mathrm{GWD} /$ & $60 \mathrm{GWD} /$ & $75 \mathrm{GWD} /$ \\
& MTU $(\mathrm{Bq} / \mathrm{g})$ & MTU $(\mathrm{Bq} / \mathrm{g})$ & MTU $(\mathrm{Bq} / \mathrm{g})$ & MTU $(\mathrm{Bq} / \mathrm{g})$ \\
\hline${ }^{125} \mathrm{Sb}$ & $8.635 \times 10^{6}$ & $1.478 \times 10^{7}$ & $1.933 \times 10^{7}$ & $2.286 \times 10^{7}$ \\
${ }^{125 \mathrm{~m}} \mathrm{Te}$ & $2.108 \times 10^{6}$ & $3.608 \times 10^{6}$ & $4.719 \times 10^{6}$ & $5.582 \times 10^{6}$ \\
${ }^{55} \mathrm{Fe}$ & $1.868 \times 10^{6}$ & $3.217 \times 10^{6}$ & $4.316 \times 10^{6}$ & $5.262 \times 10^{6}$ \\
${ }^{119 \mathrm{~m}} \mathrm{Sn}$ & $2.428 \times 10^{5}$ & $3.149 \times 10^{5}$ & $3.582 \times 10^{5}$ & $3.946 \times 10^{5}$ \\
${ }^{121 \mathrm{~m}} \mathrm{Sn}$ & $6.243 \times 10^{4}$ & $1.286 \times 10^{5}$ & $1.968 \times 10^{5}$ & $2.658 \times 10^{5}$ \\
${ }^{121} \mathrm{Sn}$ & $4.845 \times 10^{4}$ & $9.981 \times 10^{4}$ & $1.527 \times 10^{5}$ & $2.063 \times 10^{5}$ \\
${ }^{93} \mathrm{Zr}$ & $1.293 \times 10^{4}$ & $2.750 \times 10^{4}$ & $4.366 \times 10^{4}$ & $6.131 \times 10^{4}$ \\
${ }^{60} \mathrm{Co}$ & $5.787 \times 10^{3}$ & $2.598 \times 10^{4}$ & $6.308 \times 10^{4}$ & $1.185 \times 10^{5}$ \\
${ }^{93 \mathrm{~m}} \mathrm{Nb}$ & $4.887 \times 10^{3}$ & $1.111 \times 10^{4}$ & $1.870 \times 10^{4}$ & $2.762 \times 10^{4}$ \\
${ }^{54} \mathrm{Mn}$ & $2.271 \times 10^{3}$ & $2.966 \times 10^{3}$ & $3.359 \times 10^{3}$ & $3.667 \times 10^{3}$ \\
${ }^{\mathrm{T}} \mathrm{Tal}$ & $1.299 \times 10^{7}$ & $2.222 \times 10^{7}$ & $2.921 \times 10^{7}$ & $3.478 \times 10^{7}$ \\
\hline
\end{tabular}

Table 3 The radioactivation calculation results of Zirlo for various burn-ups of 30, 45, 60, and 75 GWD/MTU (only major nuclides are shown)

\begin{tabular}{lllll}
\hline & $30 \mathrm{GWD} /$ & $45 \mathrm{GWD} /$ & $60 \mathrm{GWD} /$ & $75 \mathrm{GWD} /$ \\
& MTU $(\mathrm{Bq} / \mathrm{g})$ & MTU $(\mathrm{Bq} / \mathrm{g})$ & MTU $(\mathrm{Bq} / \mathrm{g})$ & MTU $(\mathrm{Bq} / \mathrm{g})$ \\
\hline${ }^{93 \mathrm{~m}} \mathrm{Nb}$ & $1.562 \times 10^{7}$ & $3.191 \times 10^{7}$ & $4.854 \times 10^{7}$ & $6.532 \times 10^{7}$ \\
${ }^{125} \mathrm{Sb}$ & $6.642 \times 10^{6}$ & $1.137 \times 10^{7}$ & $1.487 \times 10^{7}$ & $1.758 \times 10^{7}$ \\
${ }^{125 \mathrm{~m}} \mathrm{Te}$ & $1.622 \times 10^{6}$ & $2.776 \times 10^{6}$ & $3.631 \times 10^{6}$ & $4.294 \times 10^{6}$ \\
${ }^{55} \mathrm{Fe}$ & $9.344 \times 10^{5}$ & $1.608 \times 10^{6}$ & $2.158 \times 10^{6}$ & $2.631 \times 10^{6}$ \\
${ }^{94} \mathrm{Nb}$ & $5.459 \times 10^{5}$ & $1.116 \times 10^{6}$ & $1.691 \times 10^{6}$ & $2.256 \times 10^{6}$ \\
${ }^{119 \mathrm{~m}} \mathrm{Sn}$ & $1.868 \times 10^{5}$ & $2.422 \times 10^{5}$ & $2.756 \times 10^{5}$ & $3.036 \times 10^{5}$ \\
${ }^{121 \mathrm{~m}} \mathrm{Sn}$ & $4.802 \times 10^{4}$ & $9.893 \times 10^{4}$ & $1.515 \times 10^{5}$ & $2.045 \times 10^{5}$ \\
${ }^{121} \mathrm{Sn}$ & $3.727 \times 10^{4}$ & $7.678 \times 10^{4}$ & $1.175 \times 10^{5}$ & $1.586 \times 10^{5}$ \\
${ }^{93} \mathrm{Zr}$ & $1.287 \times 10^{4}$ & $2.736 \times 10^{4}$ & $4.345 \times 10^{4}$ & $6.101 \times 10^{4}$ \\
${ }^{60} \mathrm{Co}$ & $2.894 \times 10^{3}$ & $1.298 \times 10^{4}$ & $3.153 \times 10^{4}$ & $5.923 \times 10^{4}$ \\
${ }^{\mathrm{T}} \mathrm{Ctal}$ & $2.564 \times 10^{7}$ & $4.925 \times 10^{7}$ & $7.151 \times 10^{7}$ & $9.288 \times 10^{7}$ \\
\hline
\end{tabular}

Table 4 The radioactivation calculation results of HANA-4 for various burn-ups of $30,45,60$, and 75 GWD/MTU (only major nuclides are shown)

\begin{tabular}{lllll}
\hline & $\begin{array}{l}30 \mathrm{GWD} / \\
\text { MTU }(\mathrm{Bq} / \mathrm{g})\end{array}$ & $\begin{array}{l}45 \mathrm{GWD} / \\
\text { MTU }(\mathrm{Bq} / \mathrm{g})\end{array}$ & $\begin{array}{l}60 \mathrm{GWD} / \\
\text { MTU }(\mathrm{Bq} / \mathrm{g})\end{array}$ & $\begin{array}{l}75 \mathrm{GWD} / \\
\text { MTU }(\mathrm{Bq} / \mathrm{g})\end{array}$ \\
\hline${ }^{93 \mathrm{~m}} \mathrm{Nb}$ & $2.341 \times 10^{7}$ & $4.787 \times 10^{7}$ & $7.281 \times 10^{7}$ & $9.519 \times 10^{7}$ \\
${ }^{125} \mathrm{Sb}$ & $2.656 \times 10^{6}$ & $4.547 \times 10^{6}$ & $5.947 \times 10^{6}$ & $6.689 \times 10^{6}$ \\
${ }^{55} \mathrm{Fe}$ & $1.868 \times 10^{6}$ & $3.217 \times 10^{6}$ & $4.316 \times 10^{6}$ & $4.999 \times 10^{6}$ \\
${ }^{94} \mathrm{Nb}$ & $8.188 \times 10^{5}$ & $1.675 \times 10^{6}$ & $2.539 \times 10^{6}$ & $3.316 \times 10^{6}$ \\
${ }^{125 \mathrm{~m}} \mathrm{Te}$ & $6.488 \times 10^{5}$ & $1.110 \times 10^{6}$ & $1.452 \times 10^{6}$ & $1.634 \times 10^{6}$ \\
${ }^{119 \mathrm{~m}} \mathrm{Sn}$ & $7.471 \times 10^{4}$ & $9.688 \times 10^{4}$ & $1.102 \times 10^{5}$ & $1.100 \times 10^{5}$ \\
${ }^{121 \mathrm{~m}} \mathrm{Sn}$ & $1.921 \times 10^{4}$ & $3.957 \times 10^{4}$ & $6.056 \times 10^{4}$ & $7.984 \times 10^{4}$ \\
${ }^{121} \mathrm{Sn}$ & $1.491 \times 10^{4}$ & $3.072 \times 10^{4}$ & $4.700 \times 10^{4}$ & $6.196 \times 10^{4}$ \\
${ }^{93} \mathrm{Zr}$ & $1.286 \times 10^{4}$ & $2.733 \times 10^{4}$ & $4.340 \times 10^{4}$ & $5.947 \times 10^{4}$ \\
${ }^{60} \mathrm{Co}$ & $5.787 \times 10^{3}$ & $2.598 \times 10^{4}$ & $6.308 \times 10^{4}$ & $1.113 \times 10^{5}$ \\
${ }^{\mathrm{T}} \mathrm{Cotal}$ & $2.954 \times 10^{7}$ & $5.864 \times 10^{7}$ & $8.738 \times 10^{7}$ & $1.123 \times 10^{8}$ \\
\hline
\end{tabular}

Table 5 The radioactivation calculation results of HANA-6 for various burn-ups of 30, 45, 60, and $75 \mathrm{GWD} / \mathrm{MTU}$ (only major nuclides are shown)

\begin{tabular}{lllll}
\hline & $\begin{array}{l}30 \mathrm{GWD} / \\
\text { MTU }(\mathrm{Bq} / \mathrm{g})\end{array}$ & $\begin{array}{l}45 \mathrm{GWD} / \\
\text { MTU }(\mathrm{Bq} / \mathrm{g})\end{array}$ & $\begin{array}{l}60 \mathrm{GWD} / \\
\text { MTU }(\mathrm{Bq} / \mathrm{g})\end{array}$ & $\begin{array}{l}75 \mathrm{GWD} / \\
\text { MTU }(\mathrm{Bq} / \mathrm{g})\end{array}$ \\
\hline${ }^{93 \mathrm{~m}} \mathrm{Nb}$ & $1.717 \times 10^{7}$ & $3.511 \times 10^{7}$ & $5.339 \times 10^{7}$ & $7.186 \times 10^{7}$ \\
${ }^{94} \mathrm{Nb}$ & $6.005 \times 10^{5}$ & $1.228 \times 10^{6}$ & $1.861 \times 10^{6}$ & $2.483 \times 10^{6}$ \\
${ }^{63} \mathrm{Ni}$ & $7.658 \times 10^{4}$ & $1.601 \times 10^{5}$ & $2.477 \times 10^{5}$ & $3.375 \times 10^{5}$ \\
${ }^{93} \mathrm{Zr}$ & $1.299 \times 10^{4}$ & $2.762 \times 10^{4}$ & $4.387 \times 10^{4}$ & $6.160 \times 10^{4}$ \\
${ }^{60} \mathrm{Co}$ & $9.138 \times 10^{3}$ & $1.726 \times 10^{4}$ & $2.444 \times 10^{4}$ & $3.078 \times 10^{4}$ \\
${ }^{90} \mathrm{Y}$ & $1.110 \times 10^{2}$ & $4.851 \times 10^{2}$ & $1.200 \times 10^{3}$ & $2.336 \times 10^{3}$ \\
${ }^{90} \mathrm{Sr}$ & $1.109 \times 10^{2}$ & $4.849 \times 10^{2}$ & $1.199 \times 10^{3}$ & $2.336 \times 10^{3}$ \\
${ }^{91} \mathrm{Nb}$ & $7.403 \times 10^{0}$ & $1.661 \times 10$ & $2.759 \times 10$ & $4.041 \times 10$ \\
${ }^{65} \mathrm{Zn}$ & $4.911 \times 10^{0}$ & $1.513 \times 10$ & $2.968 \times 10$ & $4.861 \times 10$ \\
${ }^{99} \mathrm{Tc}$ & $8.250 \times 10^{-1}$ & 7.693 & $2.971 \times 10$ & $7.920 \times 10$ \\
${ }^{\mathrm{T} o t a l}$ & $1.787 \times 10^{7}$ & $3.655 \times 10^{7}$ & $5.558 \times 10^{7}$ & $7.477 \times 10^{7}$ \\
\hline & & & &
\end{tabular}
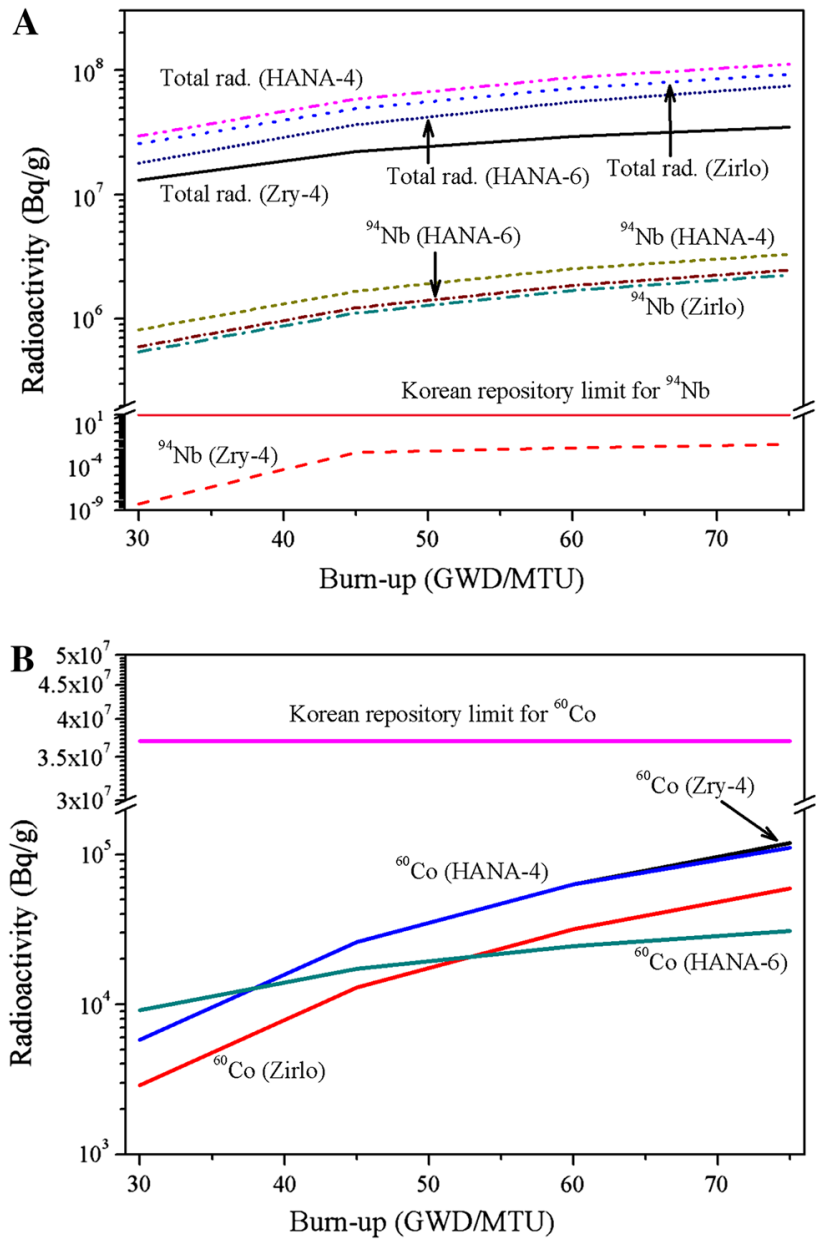

Fig. 1 Comparison of radioactivity of (a) ${ }^{94} \mathrm{Nb}$ and (b) ${ }^{60} \mathrm{Co}$ for various cladding materials and fuel burn-ups 